\title{
Mechanisms and Dynamics of Cortical Motor Inhibition in the Stop-signal Paradigm: A TMS Study
}

\author{
Wery P. M. van den Wildenberg ${ }^{1}$, Borís Burle ${ }^{2}$, Franck Vidal ${ }^{2}$, \\ Maurits W. van der Molen ${ }^{1}$, K. Richard Ridderinkhof ${ }^{1}$, \\ and Thierry Hasbroucq ${ }^{2}$
}

\begin{abstract}
The ability to stop ongoing motor responses in a splitsecond is a vital element of human cognitive control and flexibility that relies in large part on prefrontal cortex. We used the stop-signal paradigm to elucidate the engagement of primary motor cortex (M1) in inhibiting an ongoing voluntary motor response. The stop-signal paradigm taps the ability to flexibly countermand ongoing voluntary behavior upon presentation of a stop signal. We applied single-pulse TMS to M1 at several intervals following the stop signal to track the time course of excitability of the motor system related to generating and stopping a manual response. Electromyography recorded from the flexor pollicis brevis allowed quantification of the excitability of the corticospinal tract and the involvement of intracortical $\mathrm{GABA}_{\mathrm{B}}$ ergic circuits within M1, indexed respectively by the am-
\end{abstract}

\section{INTRODUCTION}

The ability to stop an ongoing voluntary response is a key aspect of cognitive control that allows people to adapt quickly to environmental changes (see Verbruggen \& Logan, 2008; Logan, 1994, for reviews). The aim of the present study is to elucidate the neural basis and the dynamics of stop-signal inhibition at the level of primary motor cortex (M1) using a novel combination of the stop-signal paradigm and TMS. The vital importance of response inhibition for adaptive behavioral interaction with the ever-changing environment is underscored by a wide range of clinical conditions characterized by inhibitory deficits, such as attention deficit hyperactivity disorder (Aron \& Poldrack, 2005; Nigg, 2005; Ridderinkhof, Scheres, Oosterlaan, \& Sergeant, 2005), Parkinson's disease (Wylie et al., 2009; Gauggel, Rieger, \& Feghoff, 2004), and obsessive-compulsive disorder (Penadés et al., 2006).

Over the years, the go/no-go task has been a popular RT paradigm to study the neurocognitive basis of response inhibition. This task requires participants to execute a

\footnotetext{
${ }^{1}$ University of Amsterdam, ${ }^{2}$ Aix-Marseille Université, CNRS, Marseille
}

plitude of the motor-evoked potential and the duration of the late part of the cortical silent period (SP). The results extend our knowledge of the neural basis of inhibitory control in three ways. First, the results revealed a dynamic interplay between response activation and stopping processes at M1 level during stop-signal inhibition of an ongoing response. Second, increased excitability of inhibitory interneurons that drives SP prolongation was evident as early as $134 \mathrm{msec}$ following the instruction to stop. Third, this pattern was followed by a stoprelated reduction of corticospinal excitability implemented around 180 after the stop signal. These findings point to the recruitment of $\mathrm{GABA}_{\mathrm{B}}$ ergic intracortical inhibitory circuits within M1 in stop-signal inhibition and support the notion of stopping as an active act of control. speeded manual response to go signals (e.g., a low tone) and to withhold any overt response upon a no-go signal (e.g., a high tone). Successful performance is thought to require the inhibition of the motor response in a no-go situation. The go/no-go task has been combined with psychophysiological techniques such as electroencephalography (Nieuwenhuis, Yeung, van den Wildenberg, \& Ridderinkhof, 2003; Eimer, 1993; Jodo \& Kayama, 1992; Kok, 1986; Pfefferbaum, Ford, Weller, \& Kopell, 1985) and functional magnetic resonance imaging. Contrasting patterns of brain activation on no-go and go trials generally reveal a predominantly right-hemispheric distributed network associated with no-go trials, that includes dorsolateral, inferior, and bilateral superior prefrontal cortices, the supplementary motor area, the anterior cingulate, inferior parietal and temporal cortices, the caudate nucleus, and the cerebellum (Simmonds, Pekar, \& Mostofsky, 2008; Kelly et al., 2004; Durston, Thomas, Worden, Yang, \& Casey, 2002; Garavan, Ross, \& Stein, 1999). Although activation in these structures correlates with no-go inhibition, changes in regional blood flow are not easily translated in terms of response-related activation or inhibition. Furthermore, the time course of the BOLD response evolves over seconds, which makes it difficult to capture the split-second 
dynamics of processes that contribute to countermanding a voluntary action.

\section{Transcranial Magnetic Stimulation of M1}

TMS allows noninvasive stimulation of the brain and has the advantage of providing accurate temporal information to investigate the neurobiology of cognitive processes. TMS involves application of a powerful, rapidly changing magnetic field to induce electric fields in cortex that may cause short-term disruption of cortical function (e.g., Chambers et al., 2006; Walsh \& Cowey, 2000). On the other hand, single-pulse TMS over M1 provides a useful tool to study the dynamics of motor excitability when subjects are engaged in a cognitive task (Reis et al., 2008; Hallett, 2007). Stimulation of cortical motor areas representing the hand that is involved in the task elicits specific electromyographic (EMG) potentials in the corresponding hand muscles that reflect the excitability of the motor system during the generation and inhibition of responses. The amplitude of the motor-evoked potential (MEP) indexes the excitability of the corticospinal tract, whereas the late part of the cortical silent period (SP) reflects the involvement of inhibitory neural circuits intrinsic to M1 (Terao \& Ugawa, 2002).

Go/no-go studies that report MEP amplitudes elicited by single-pulse TMS of M1 show enhanced corticospinal excitability after go stimuli, whereas processing of no-go stimuli is associated with a suppression of corticospinal excitability, both evolving about $150 \mathrm{msec}$ after signal presentation (Leocani, Cohen, Wassermann, Ikoma, \& Hallett, 2000; Hoshiyama et al., 1996, 1997). Hoshiyama and colleagues concluded that movement prevention is associated with a strong nonspecific inhibition of the pyramidal tract (see also Nakata et al., 2006; Leocani et al., 2000). MEPs elicited by a single TMS pulse are a compound measure that reflects both cortical and downstream (spinal) processing and, therefore, do not isolate intracortical changes in motor excitability. Conversely, the specific involvement of short-interval intracortical inhibition mechanisms (SICI) at M1 can be assessed by paired pulse techniques (Kujirai et al., 1993). Here, a conditioning stimulus (CS) recruits inhibitory interneurons that modulate the MEP amplitude produced by the test stimulus (TS). Paired-pulse TMS at short interstimulus intervals (1-6 msec) allows the assessment of $\mathrm{GABA}_{\mathrm{A}}$ ergic SICI, whereas longer intervals in the range of 50-200 msec test the involvement of $\mathrm{GABA}_{\mathrm{B}}$ ergic long-interval intracortical inhibition (LICI; see Chen et al., 1994). GABA receptors also mediate the duration of the late cortical SP (Werhahn, 1999). In no-go situations, MEP amplitudes elicited by a TS following $2 \mathrm{msec}$ after a CS are significantly reduced compared to a control situation (Sohn, Wiltz, \& Hallett, 2002; Waldvogel et al., 2000), reflecting the recruitment of intracortical $\mathrm{GABA}_{\mathrm{A}}$ ergic inhibitory interneurons when preventing a movement (Ziemann, Lonnecker, Steinhoff, \& Paulus, 1996). Next to enhanced
SICI, Sohn et al. (2002) also observed a no-go related reduction in LICI using longer TMS intervals of $80 \mathrm{msec}$, reflected by increased conditional MEP amplitudes. Because LICI is known to inhibit SICI, the no-go related increase in SICI reported by Sohn et al. might be partially due to the observed decrease in LICI (Sanger, Garg, \& Chen, 2001).

The application of single-pulse and paired-pulse TMS in combination with the go/no-go paradigm has provided new information about motor-related processes that underlie the prevention of generating voluntary movements. In the no-go tasks discussed above, go and no-go signals were presented with equal proportions (Sohn et al., 2002; Waldvogel et al., 2000; Hoshiyama et al., 1997). It has been noticed that this particular setup might be considered suboptimal for inducing preactivation of the go response (e.g., Nakata et al., 2005; Kok, Ramautar, de Ruiter, \& Ridderinkhof, 2004; Casey et al., 2001). Other paradigms have been introduced that arguably involve stronger response activation, and thus, offer a more suitable opportunity to study the inhibition of ongoing actions. These alternative paradigms will be outlined briefly in the next sections.

\section{The Movement-prevention Paradigm}

The movement-prevention paradigm (Slater-Hammel, 1960) allows the examination of inhibitory processing during the cancellation of movement initiation rather than inhibition of an ongoing response to an imperative go stimulus. In a recent study, Coxon, Stinear, and Byblow (2006, Experiment 2) tracked the involvement of M1 excitability by presenting a single subthreshold TMS pulse at several intervals following a movement-prevention signal. The task required participants to lift a finger from a keyboard key to stop a rotating clock dial at a stationary target (i.e., the number 8 ). On 33\% of the trials, the sweep dial could stop before it reached the target, upon which the participant should refrain from moving their finger. Initiation of a finger lift was characterized by enhanced corticomotor excitability, indexed by increased MEP amplitudes following subthreshold TMS (Coxon et al., 2006, see also Chen, Yaseen, Cohen, \& Hallett, 1998; Rossini, Zarola, Stalberg, \& Caramia, 1988). This increase was observed in index finger muscles involved in the task, but was absent in EMG recorded from proximate but noninvolved thumb muscles. Interestingly, MEP amplitudes on trials on which participants kept their finger depressed showed a reduction relative to go trials, starting about 140 msec after the movement-prevention signal. This reduced excitability recorded over the involved hand muscles was paralleled by a significant suppression of excitability recorded over noninvolved muscles, evolving 160 msec after the cue, which points to the nonspecific nature of response inhibition.

Using the same Slater-Hammel paradigm, Coxon and coworkers (Experiment 3) also applied paired-pulse stim- 
ulation with CS and TS separated by 2.5 msec. TMS was presented 150 msec after the onset of the movementprevention cue and yielded a reduced MEP to the TS pulse during the prevention of a movement as compared to go trials. This finding points to the increased excitability of $\mathrm{GABA}_{\mathrm{A}}$ ergic intracortical inhibition circuits operating at M1 when refraining from initiating a movement. Detailed temporal information on stop-cue related recruitment of intracortical inhibition circuits is not yet available, as TMS was applied at one fixed interval in available paired-pulse studies (Coxon et al., 2006; Sohn et al., 2002; Waldvogel et al., 2000).

\section{The Present Study}

TMS procedures have provided new insights into the involvement and the temporal processing dynamics of M1 in no-go situations (Sohn et al., 2002; Leocani, 2000; Waldvogel et al., 2000; Hoshiyama et al., 1996, 1997) and when canceling the initiation of a response (Coxon et al., 2006; see Stinear, Coxon, \& Byblow, 2009 for a review). Here we apply single-pulse TMS of M1 in the context of the stop-signal paradigm, an increasingly popular task to assess the efficiency of inhibitory control over prepotent ongoing voluntary responses (Logan, 1994; Logan \& Cowan, 1984). In the stop task, subjects usually perform a speeded response to a visual stimulus, the go signal. On some trials, the onset of the go signal is followed shortly by a stop signal that instructs the participant to withhold the button-press response. Information processing on stop trials has been formally conceptualized in terms of a race between go and stop processes (Logan \& Cowan, 1984). The process that finishes first determines whether the response is executed or inhibited. If the go process initiated by the go signal finishes first, the motor response will be executed. Conversely, if the stop process wins the race, the go response will be countermanded. Given the assumptions of the race model, it is possible to estimate the latency of the stop process, or stop-signal RT (SSRT; Logan \& Cowan, 1984; see also Band, van der Molen, \& Logan, 2003). With SSRT as an index of inhibitory efficiency, the stop-signal paradigm provides advantages that supplement go/no-go procedures that have been employed to assess response inhibition. Note that the design of the stop task differs from that of the SlaterHammel paradigm (Coxon, Stinear, \& Byblow, 2007; Coxon et al., 2006; Slater-Hammel, 1960). In the stop task, participants are occasionally presented with a stop signal after the imperative go signal, whereas in the movement-prevention task, the stop cue prevents initiation of a prepared response. In the latter situation, initiation of a prepared movement is aborted, whereas the stop task requires stopping of an already signaled response process.

The stop task is becoming one of the golden standards in assessing stopping efficiency, representative for dailylive instances of response inhibition (e.g., in traffic) and has been used extensively to study inhibitory control over a wide variety of actions in nonclinical (e.g., Williams, Ponesse, Schachar, Logan, \& Tannock, 1999) as well as in clinical samples, such as individuals diagnosed with attention deficit/hyperactivity disorder (Schachar \& Logan, 1990), patients with damage to prefrontal cortex (Aron \& Poldrack, 2003), and drug-using individuals (Colzato, van den Wildenberg, \& Hommel, 2007; Fillmore \& Rush, 2002). Stop-signal inhibition relies in large part on prefrontal cortex (Chambers et al., 2006; Aron, Robbins, \&, Poldrack, 2004; Aron, Fletcher, Bullmore, Sahakian, \& Robbins, 2003; Band \& van Boxtel, 1999), in interaction with other brain regions such as the basal ganglia (Aron \& Poldrack, 2006; van den Wildenberg et al., 2006; Casey, Tottenham, \& Fossella, 2002). Recent neuroimaging work on the neural correlates of stop-signal inhibition by Aron et al. revealed a densely interconnected neuronal network that includes right inferior frontal cortex (rIFC), right presupplementary motor area, and subthalamic nucleus. Activation in these circuits was shown to covary with individual differences in the efficiency of stopping a prepotent response as indexed by SSRT (Aron, Behrens, Smith, Frank, \& Poldrack, 2007). These regions seem to differ from brain areas that have been related to performance on no-go trials, which points to processing differences between stop-signal and go/no-go paradigms (see also Rubia et al., 2001).

To elucidate the role of M1 in the inhibition of ongoing voluntary responses, we combined the stop-task with single-pulse TMS. Presenting TMS at variable intervals following go and stop signals allowed tracking the temporal dynamics of cortical and corticospinal excitability related to responding and stopping. Peak-to-peak amplitudes of the MEP provide information about the excitability of the corticospinal tract, whereas the late part of the cortical SP represents the involvement of intracortical inhibition by $\mathrm{GABA}_{\mathrm{B}}$ ergic neural circuits intrinsic to M1 (Terao \& Ugawa, 2002; Schnitzler \& Benecke, 1994). We also compared the temporal dynamics of motor activation on trials where participants were able to stop their response with trials on which inhibition failed. Furthermore, this study is the first to track the excitability of intracortical inhibition circuits during stop-signal processing over multiple time points.

\section{METHODS}

\section{Participants}

Eight healthy volunteers (2 women, $M=33$ years of age, $S D=9$ years), with no relevant psychiatric or neurological history, participated in the experiment. They were all right-handed and had normal or corrected-to-normal vision. Written informed consent was obtained from each individual prior to participation. This study was performed in accordance with the Declaration of Helsinki principles and has been approved by the local ethics committee. 


\section{Apparatus and Signals}

Subjects were tested in a darkened room while seated in a comfortable chair that supported hands and arms. A vacuum-controlled head support (model no. 155, Burnett Body Support, RBF Healthcare, Essex, UK) maintained a light and comfortable restraint of the participant's head. With the right hand, the participant gripped a plastic cylinder $(3 \mathrm{~cm}$ diameter, $11 \mathrm{~cm}$ in height) that was fixed on a pullout table. The distal phalanx of the right thumb rested on a force sensor (model ELM-16A-100, Entran Devices, Fairfield, NJ) on top of the cylinder. The experiment was controlled by a microcomputer. Subjects faced a black panel, placed $1 \mathrm{~m}$ in front at eye level, which held three light-emitting diodes that were positioned on a vertical line (see Figure 1). The upper and lower diodes conveyed information about the force window (10-20 N), that is, the range of isometric precontraction force that was required to initiate a trial (see also Burle, Bonnet, Vidal, Possamai, \& Hasbroucq, 2002). This self-initiation procedure generated the background EMG activity necessary for visualizing the SP. The lower diode lit up blue when the precontraction force entered the force window, crossing the lower bound of $10 \mathrm{~N}$. If the precontraction force was too low and dropped below this value within 1 sec, the lower diode extinguished and the trial was paused until the subject re-entered the force window. The upper diode lit up blue if subjects pressed too hard and the precontraction force crossed the upper bound of the force window fixed at $20 \mathrm{~N}$. If so, the experiment was paused and participants had to release the force button, upon which the upper diode extinguished, in order to re-enter the force window.

When the precontraction force had been maintained between 10 and $20 \mathrm{~N}$ for $1 \mathrm{sec}$, the central bicolor diode conveyed the imperative signal by turning green or red. We chose not to vary the foreperiod within a block of

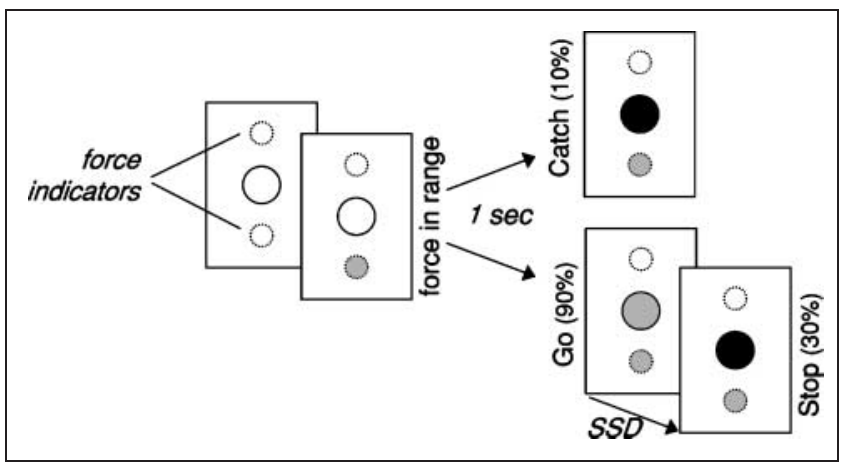

Figure 1. The lower force indicator lit up when force crossed the lower bound of the force window (i.e., $10 \mathrm{~N}$ ). The upper force indicator lit up if force exceeded $20 \mathrm{~N}$, after which the trial was aborted. If force was maintained between 10 and $20 \mathrm{~N}$ for $1 \mathrm{sec}$, the central LED conveyed either a red catch signal (10\%) or a green go signal (90\%) of which 30\% turned red after a variable stop-signal delay (SSD) indicating a stop signal. trials because longer foreperiods yield longer SSRTs ( $\mathrm{Li}$, Krystal, \& Mathalon, 2005). This positive correlation suggests that subjects' readiness to respond to the go signal interacts with stopping latency. To avoid this systematic response bias, we used a fixed duration of $1 \mathrm{sec}$. This setup, however, might result in enhanced response readiness reflected by large numbers of stop-respond trials and short stop-signal delays. This was avoided by presenting $10 \%$ catch trials (i.e., 10\%) to reduce anticipation of the presentation of a go signal.

Signals were response-terminated or terminated after 1500 msec if no response was given. Responses consisted of a firm press with the right thumb on the force sensor. RT was defined as the moment at which the response force exceeded $40 \mathrm{~N}$. A brief tone $(1600 \mathrm{~Hz}, 75 \mathrm{~dB}$, $200 \mathrm{msec}$ ) marked this event to inform the subject that the response was forceful enough. The same auditory signal indicated the end of the intertrial interval (either 2000, 2250, 2500, 2750, or $3000 \mathrm{msec}$, mixed randomly), instructing the subject to enter the force window to initiate the next trial.

\section{Task and Procedure}

Participants performed a stop task. They were instructed to press a force transducer with the thumb of the dominant right hand if the central diode turned green (go trial). The percentage of go signals was 90\%. Subjects had to refrain from responding if the central diode lit up red, indicating a catch signal (10\%). Go and catch trials were randomly intermixed. Only behavioral data were analyzed for catch trials.

A visual stop signal shortly followed $30 \%$ of the go signals. The stop cue was an unpredictable color change of the central diode from green to red. Participants were instructed to refrain from responding upon a stop signal. A dynamic tracking algorithm continuously adjusted stopsignal delay, that is, the interval between the presentation of the stop signal relative to the onset of the go signal (Levitt, 1971). If stopping was successful on two consecutive stop trials, stop-signal delay on the next stop trial was increased by $25 \mathrm{msec}$. Failures to inhibit were followed by a 25 -msec decrease in stop-signal delay. This procedure biased the chances of successful inhibition which theoretically converges to $71.4 \%$ correctly inhibited stop trials. This bias was introduced in order to obtain sufficient trial numbers for computing reliable averages of MEP and SP scores related to stopping.

Task instructions stressed the importance of responding fast to green diodes while premature responses were discouraged. Subjects were told not to respond to catch signals and to try to stop the button press when the color of the central green diode changed to red. It was explained to them that stop-signal delay varied so that on some trials stopping would be easier than on other trials. The stop task was practiced in four training blocks of 100 trials each to familiarize the subject with the task and 
the self-initializing procedure. The training blocks were followed by two calibration blocks of 100 trials each in order to obtain individual distributions of go RTs and SSRT values. These data were used to set the timing of TMS for each individual in the experimental sessions. The experiment consisted of a TMS session and a control session. The TMS session contained 12 blocks of 100 trials and was completed in about $3 \mathrm{hr}$.

Eliciting a magnetic pulse $(<1 \mathrm{msec})$ causes the coil to produce a brief sound with an amplitude that depends on stimulation intensity. The sound generated by the MagStim200 unit used in the present study resembles a click. It is well known that a (task-irrelevant) auditory signal presented about the same time as a visual response signal facilitates RT (van der Molen \& Keuss, 1979; Nickerson, 1973). Thus, the effects of TMS on performance are a combination of effects resulting from both magnetic and acoustic stimulation. To determine the effects of TMS on performance, it is therefore necessary to take into account possible effects of the accompanying acoustic stimulation. Therefore, the coil was reversed in a control session such that the induced current was flowing in the anterior-posterior direction (i.e., opposite to the direction in the TMS session). Although inhibitory interneurons have a lower threshold than excitatory interneurons (Davey, Romaiguère, Maskill, \& Ellaway, 1994), the absence of reliable MEP and SP traces verified that there were no EMG effects in the control condition.

The control session comprised six blocks of 100 trials, taking approximately $1.5 \mathrm{hr}$ to complete. Half the participants started with the TMS session and then completed the control session; the other half performed the experiment in the reversed order. TMS and control sessions were performed on separate days.

\section{Transcranial Magnetic Stimulation}

\section{TMS Procedure}

The coil was held in a swan neck that was bolted on the back of the chair. Magnetic stimulation was delivered using a figure eight shaped coil with a $7-\mathrm{cm}$ diameter connected to a MagStim200 unit (Magstim, Whitland, UK) with $2.2 \mathrm{~T}$ maximum output intensity (stimulus duration $<1 \mathrm{msec})$. The coil was placed tangentially on the head over left M1. The coil was first centered over primary motor cortex of the right hand, approximately $5.5 \mathrm{~cm}$ to the left of the vertex (Cz, 10/20 Electrode System, Jasper, 1958). Next, the coil was moved in the anterior-posterior and coronal directions until the lowest threshold spot for activating the right flexor pollicis brevis was reached (see also Davranche et al., 2007). In the TMS session, the induced current was flowing in posterior-anterior direction over the cortical hand representation. The stimulation intensity range was set individually using a computer-controlled series of tuning trials to adjust the intensity of TMS stimulation for each partic- ipant. During each series of adjustment trials, the participant exerted a constant press (between 10 and $20 \mathrm{~N}$ ) on the sensors while receiving 20 stimulations delivered in a pseudorandom temporal sequence. The EMG signal averaged over the trials and time-locked to the occurrence of the stimulation was displayed on the computer screen to be visually examined. TMS intensity was adjusted to the minimal intensity to produce a peak-to-peak MEP of about $100 \mu \mathrm{V}$ on the trace averaged over the 20 adjustment trials and set 5\% above this value for the experiment. Individual stimulation intensity was kept constant throughout the experiment, all within a range of $30 \%$ to $47 \%$ of maximum output intensity for the subjects tested ( $M=39 \%$; see Table 1$)$.

\section{TMS Timing}

To capture the dynamics of go and stop-signal processing, we applied TMS at various intervals with respect to the onset of the go signal and the stop signal. The number of TMS intervals was calculated with consideration of (1) a sufficient number of observations in order to obtain reliable MEP and SP averages per TMS interval, and (2) maximal coverage of the entire process, that is, from go-signal onset to go RT and from stop-signal onset to SSRT. The time period in between go-signal onset and go $\mathrm{RT}$ is longer than the time period in between stop-signal onset and SSRT (roughly 1.5 times as long), Therefore, based on the above considerations, there were 1.5 times as many TMS intervals on go than on stop trials. This resulted in six equidistant TMS intervals on go trials and four equidistant intervals on stop trials. The behavioral data obtained in the calibration blocks were used to determine the TMS intervals in the experimental sessions (see Table 1). TMS was delivered on $50 \%$ of the go trials, at one of six equidistant time points (i.e., at 14\%, 29\%, 43\%, $57 \%, 71 \%$, and $86 \%$ ) dividing the interval between gosignal onset and the value determined by the first decile of the go RT distribution recorded in the calibration session (see Figure 2). This procedure resulted in 63 go trials per TMS interval. TMS was applied at 35, 69, 104, 139, 174, and $208 \mathrm{msec}$ (indicated as goT1 to goT6) after the go signal (see Table 1). TMS was delivered at 50 msec or at $150 \mathrm{msec}(p=.5)$ after the onset of catch signals to keep these trials similar to go and stop trials in terms of occasional TMS stimulation. On stop trials, TMS was applied at one of four equidistant time points (i.e., 20\%, $40 \%, 60 \%$, and $80 \%$ of the SSRT) dividing the interval between stop-signal onset and individual SSRT computed over the calibration session. ${ }^{1}$ This yielded 81 stop trials per TMS interval sT1, sT2, sT3, and sT4, respectively at 45, 89, 134, and $179 \mathrm{msec}$ following the stop signal. With the tracking algorithm ensuring successful inhibition on about $71 \%$ of the stop trials, approximately 57 successfully inhibited stop trials were obtained per TMS interval. 
Table 1. Age (Years), TMS Intensity (in \% Maximum Output), First RT Decile, and TMS Intervals on Go Trials (goT1 to goT6) and on Stop Trials (sT1 to sT4) per Participant

\begin{tabular}{|c|c|c|c|c|c|c|c|c|c|c|c|c|c|}
\hline \multirow[b]{3}{*}{ Subject } & \multirow[b]{3}{*}{ Age } & \multirow[b]{3}{*}{ TMS Output (\%) } & \multirow[b]{3}{*}{ 1st RT decile } & \multicolumn{10}{|c|}{ TMS Timing } \\
\hline & & & & \multicolumn{6}{|c|}{ Go Trials } & \multicolumn{4}{|c|}{ Stop Trials } \\
\hline & & & & goT1 & got2 & got3 & goT4 & goT5 & got6 & $s T 1$ & $s T 2$ & sT3 & sT4 \\
\hline 1 & 31 & 45 & 225 & 32 & 64 & 97 & 129 & 161 & 193 & 45 & 90 & 136 & 181 \\
\hline 2 & 28 & 30 & 210 & 30 & 60 & 90 & 120 & 150 & 180 & 43 & 86 & 130 & 173 \\
\hline 3 & 31 & 40 & 227 & 32 & 65 & 97 & 130 & 162 & 195 & 44 & 89 & 133 & 178 \\
\hline 4 & 45 & 38 & 299 & 43 & 83 & 128 & 171 & 213 & 256 & 47 & 94 & 141 & 188 \\
\hline 5 & 28 & 36 & 267 & 38 & 76 & 114 & 153 & 191 & 229 & 44 & 87 & 131 & 174 \\
\hline 6 & 50 & 42 & 262 & 33 & 65 & 98 & 131 & 163 & 196 & 45 & 90 & 135 & 180 \\
\hline 7 & 30 & 47 & 229 & 32 & 65 & 97 & 130 & 162 & 194 & 45 & 91 & 136 & 181 \\
\hline 8 & 23 & 37 & 227 & 37 & 75 & 112 & 150 & 187 & 224 & 43 & 87 & 130 & 174 \\
\hline Mean & 33 & 39 & 243 & 35 & 69 & 104 & 139 & 174 & 208 & 45 & 89 & 134 & 179 \\
\hline
\end{tabular}

\section{Force and EMG Recordings}

The subjects positioned the distal phalanx of the right thumb on the force sensor. Both arms rested on the table as comfortably as possible. The response was an isometric press of the right thumb on the sensor exceeding $40 \mathrm{~N}$ and was measured as a force signal, digitized on-line at $2 \mathrm{kHz}$. The EMG activity of the flexor pollicis brevis of the right hand was recorded with two $\mathrm{Ag}-\mathrm{AgCl}$ electrodes (Vickers Medical, Medelec, Woking, UK), $6 \mathrm{~mm}$ in diameter, glued $2 \mathrm{~cm}$ apart on the skin of the thenar eminence. The EMG signal was band-pass filtered between $10 \mathrm{~Hz}$ and $1 \mathrm{kHz}$ (Grass Instrument Division, West Warwick, RI, USA), and amplified (gain $\times 10,000)$ to visualize the SP and digitized on-line at $2 \mathrm{kHz}$.

EMG was recorded during $1500 \mathrm{msec}$, starting $100 \mathrm{msec}$ before trial onset. EMG activity and force signals were stored for off-line analyses with the EMG signal aligned to the onset of the imperative signal. Visual examination of the traces allowed the rejection of trials on which TMS was delivered during EMG activity that is associated with the button press (9.9\%) to avoid spurious enhancement of the MEP. The MEPs evoked during the calibration blocks served to define the time window in which the peak-to-peak value of the MEP was computed on each stimulation trial. The duration of the SP was measured in milliseconds relative to TMS onset. The end of the SP was determined visually and marked with the computer mouse as the moment of the very first occurrence of background EMG activity (Davranche et al., 2007; Fuhr, Cohen, Roth, \& Hallett, 1991). This was done by a single examiner who was blind to stimulation interval and trial information. We marked the end of the late part of the SP, ranging from 77 to 95 msec which is sufficiently long to reflect the late part of the cortical SP (see Inghilleri, Berardelli, Cruccu, \& Manfredi, 1993). Trials on which the SP was interrupted by EMG related to the button press response were rejected (22.9\%) to avoid the problem that short RTs reduce the SP, whereas long RTs allow a longer SP, inducing an artifactual correlation.
Figure 2. The RT distribution represents the finishing times of the go response process. Given that inhibition failed on $29 \%$ of the stop trials, as controlled by the tracking algorithm, SSRT is the cutoff point (e.g., $275 \mathrm{msec}$ ) minus mean stop-signal delay (SSD, e.g., $75 \mathrm{msec})=200 \mathrm{msec}$. On average, TMS followed the go signal at six intervals (black arrows) before the first go-RT decile (indicated by an $x$ ). TMS on stop trials was presented at four intervals (gray arrows) between stop-signal onset (S) and SSRT.

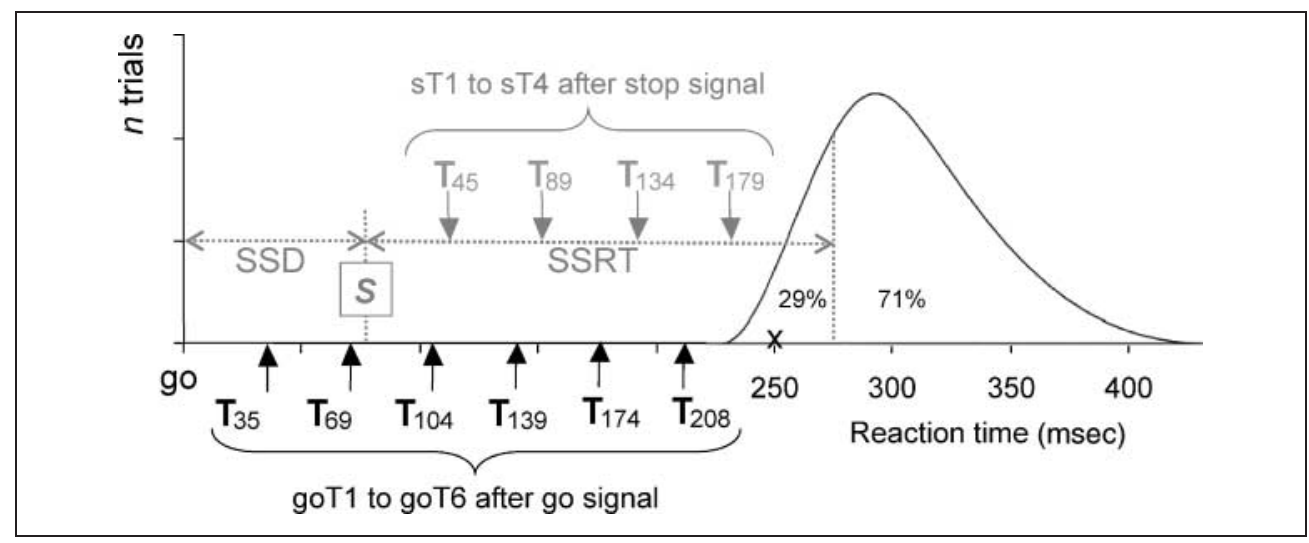




\section{Data Reduction and Analysis}

\section{Behavioral Data}

Individual mean RTs on correct trials were calculated. SSRT was computed using the procedure derived from the race model (Logan \& Cowan, 1984). Following the race model assumption of independence, the RT distribution of the go process is the same whether or not a stop signal is presented. This implies that the left side of the distribution of RTs on go-signal trials, representing fast RTs, matches the distribution of RTs on stop trials that escape inhibition (see Figure 2). The latency of the stop process can be computed from the start and the finish of the stop process (for a detailed exposition, see Logan, 1994). The start of the stop process was experimentally controlled by adjusting the stop-signal delay, but the finish time has to be inferred from the observed go RT distribution. Because TMS can influence late processing stages of the reaction process, this distribution included only RTs to go signals without TMS. If responses were not stopped on any observed $n \%$ of the stop trials, the finish of the stop process should be equal to the $n$th percentile of the RT distribution on go trials. In the present study, the tracking algorithm converged toward 29\% of the stop trials containing a response. Finally, mean stop-signal delay is subtracted from this finish time to obtain an estimate of SSRT. Stop trials on which inhibition was successful are called stop-inhibit trials, whereas stop trials on which subjects were unable to stop their response are referred to as stop-respond trials.

\section{EMG Data}

Because of large intersubject variability, individual MEP data were converted into $Z$-scores, based on individual means and standard deviations computed over all stimulation trials (see also Davranche et al., 2007). The mean and the standard deviation of the MEP amplitudes were computed per subject, regardless of TMS interval and trial type. Thereafter, the $Z$-score was computed for each individual MEP. The mean $Z$-scores were then averaged separately for each TMS interval and for each trial type. Therefore, all subjects had the same weight in the subsequent analyses. For the same reasons, the raw SP durations were $Z$-transformed in the same way as the MEPs.

\section{Statistical Tests}

Behavior on go trials (mean go RT and omission rates), on no-go trials (commission rates), and on stop trials (percentage correctly inhibited stop trials and SSRT) was analyzed using repeated measures ANOVAs with session (control session vs. TMS session), pulse (pulse vs. no-pulse trials), and TMS interval as within-subject variables. Percentages are not normally distributed and were therefore squarerooted before entered into the ANOVA. Mean $Z$-scores of MEP amplitude (mV) and SP duration (msec) were analyzed using repeated measures ANOVA with trial (go, stop-inhibit, and stop-respond trial) and TMS interval as within-subject variables. TMS was delivered at one of six intervals after go-signal onset, indicated as goT1 to goT6, and at one of four intervals after stop-signal onset, sT1 to sT4 (see Table 1). The significance level was set at $p<.05$.

\section{RESULTS}

\section{Behavioral Data}

\section{Go Trials}

Figure 3 shows mean RT on go trials with and without a pulse obtained in the TMS session and in the control session. Overall, omission rates to go signals were low and did not distinguish between the TMS session $(M=$ $0.5 \%, S E=0.1 \%)$ and the control session $(M=0.3 \%, S E=$ $0.1 \%)[F(1,7)=2.19, p=.18]$. Generally, the amount of go omissions was not affected by the presence of a pulse $(M=0.4 \%, S E=0.1 \%$, no-pulse trial vs. $M=0.5 \%, S E=$ $0.1 \%$, pulse trial, $F<1$ ). There was no significant interaction between these two factors $[F(1,7)=1.04, p=$ .34]. Finally, omission rates in both sessions did not vary with TMS interval $(F \mathrm{~S}<1)$.

Overall, RTs to go signals were comparable between the TMS session $(M=345 \mathrm{msec}, S E=17 \mathrm{msec})$ and the control session $(M=341 \mathrm{msec}, S E=16 \mathrm{msec})[F(1$, $7)=1.01, p=.35]$. Likewise, go RT on pulse trials $(M=$ $346 \mathrm{msec}, S E=17 \mathrm{msec}$ ) was comparable to RT on nopulse trials $(340 \mathrm{msec}, S E=15 \mathrm{msec})[F(1,7)=3.12$, $p=.12]$. The factors session and pulse did not interact $(F<1)$. An overall analysis conducted over both sessions

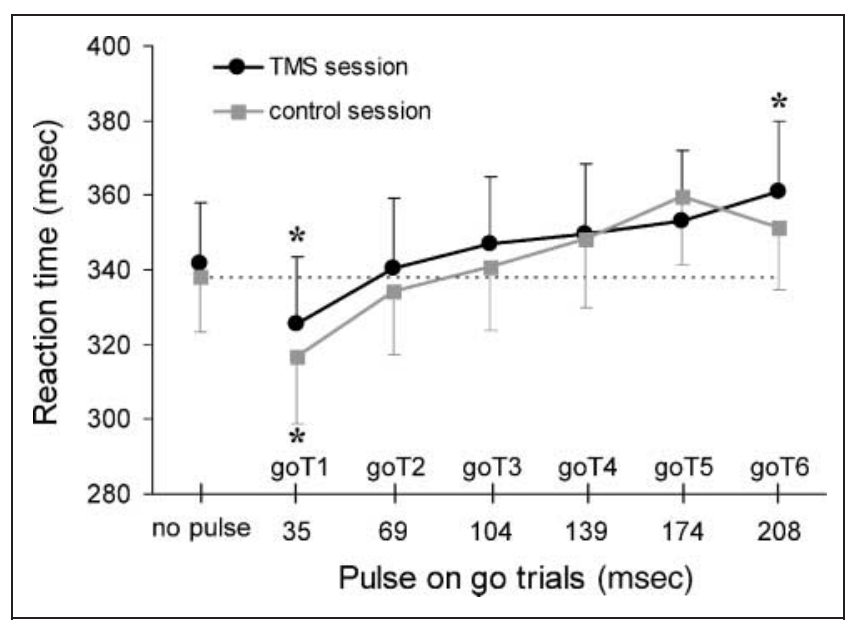

Figure 3. Mean go RT as a function of pulse (pulse trial vs. no-pulse trial) and TMS interval in the TMS session (black line) and the control session (gray line). The isolated points represent go RT on no-pulse trials. The facilitatory effect of a pulse at $35 \mathrm{msec}$ on go RT was absent at longer intervals. In the TMS session, a pulse at 208 msec prolonged go RT relative to no-pulse trials $(* p<.05)$. 
confirmed that go RT increased with TMS interval $[F(5$, $35)=15.27, p<.001]$. The lack of an interaction between session and TMS interval indicated that the linear trend was shared by the control session and the TMS session $(F<1)$. The effects of a pulse on go RT was threefold. First, a pulse at $35 \mathrm{msec}$ (i.e., the shortest interval) decreased go RT compared to RT on no-pulse trials in both the control (no-pulse vs. goT1, $p=.038$ ) and the TMS session (no-pulse vs. goT1, $p=.009$ ). This indicates that the air- and bone-conducted click evoked by the magnetic coil exerted a facilitatory effect on go RT, but only if the pulse followed shortly after the go signal. Second, TMS at intervals goT2 to goT5 did not affect go RT compared to no-pulse trials (all $p s>$.13). Third, relative to RT on nopulse trials, a pulse at $208 \mathrm{msec}$, the longest interval, significantly prolonged responding in the TMS session $(p=$ $.007)$, but not in the control session $(p=.15)$. This indicates that the application of TMS of M1 close in time to the RT delays response-related processing (see also Burle et al., 2002).

\section{Stop Trials}

The tracking algorithm worked well and obtained inhibition percentages on stop trials of $71 \%$ in both sessions, as anticipated (see Table 2). According to the race model, responses on stop trials that escaped inhibition were faster than responses on go trials $(p<.001$ for both sessions). SSRT did not differ between the control session $(M=$ $234 \mathrm{msec}, S E=5.1 \mathrm{msec})$ and the TMS session $(M=$ $239 \mathrm{msec}, S E=3.4 \mathrm{msec})[F(1,7)=1.37, p=.28]$. This finding indicates that TMS did not influence the latency of the stopping process. It is important to note that TMS over motor cortex can thus be used to probe processes of response activation and inhibition without itself interfering with these processes.
Catch Trials

Commission rates on catch trials were low. Overall, TMS interval did not affect errors on catch trials $(M=2.8 \%$, $S E=0.6 \%$ for T1 vs. $M=1.8 \%, S E=0.9 \%$ for T2) $[F(1$, $7)=1.97, p=.20]$. There was a trend toward committing more responses in the TMS session $(M=2.9 \%, S E=0.9 \%)$ than in the control session $(M=1.7 \%, S E=0.6 \%)[F(1$, 7) $=4.51, p=.07]$.

\section{MEP Amplitude}

\section{Go Trials}

Figure 4A (thick gray line) shows the averaged MEP amplitudes with the TMS intervals aligned to the onset of the go signal. A repeated measures ANOVA yielded a significant main effect of TMS interval on MEP amplitude $[F(5$, $35)=15.05, p<.0001]$. Tested directly, the MEP values did not differ between early stimulation, goT1 and goT2 $[t(7)=1.36, p=.22]$. With later stimulation, that is, from goT2 up to goT6, MEP amplitude increased in a linear fashion $[F(1,7)=54.29, p<.001]$. Table 3 presents the MEP values in millivolts.

\section{Stop Trials}

The MEP amplitudes on stop-inhibit trials (see Figure 4A, black line) were analyzed at four TMS intervals. Importantly, successful inhibition was characterized by a typical MEP pattern $[F(3,21)=18.31, p<.001]$. Pairwise comparisons (all Bonferroni corrected) confirmed that shortly after the presentation of the stop signal, MEP amplitude steadily increased up to sT3 (sT1 vs. sT2, $p=.03$, and sT2 vs. sT3, $p=.002$ ). Notably, at the last moment of stimulation (sT4 at 179 after stop-signal onset), the initial increase is followed by a sharp decline in MEP amplitude

Table 2. Mean Go RT on No-Pulse and Pulse Trials, Percentage Stop-Inhibit Trials (\%S-I), Mean Stop-Respond RT (SRRT) and Stop-Signal RT (SSRT) per Participant in the Calibration Session, the Control Session, and the TMS Session

\begin{tabular}{|c|c|c|c|c|c|c|c|c|c|c|c|c|c|c|}
\hline \multirow[b]{3}{*}{ Subject } & & & & & \multicolumn{5}{|c|}{ Control Session } & \multicolumn{5}{|c|}{ TMS Session } \\
\hline & \multicolumn{4}{|c|}{ Calibration Session } & No Pulse & \multicolumn{4}{|c|}{ Pulse } & \multirow{2}{*}{$\frac{\text { No Pulse }}{\text { Go RT }}$} & \multicolumn{4}{|c|}{ Pulse } \\
\hline & Go $R T$ & $\% S-I$ & SRRT & SSRT & Go $R T$ & Go $R T$ & $\% S-I$ & SRRT & SSRT & & Go $R T$ & $\% S-I$ & SRRT & SSRT \\
\hline 1 & 285 & 69 & 251 & 226 & 306 & 317 & 71 & 290 & 230 & 323 & 319 & 71 & 294 & 239 \\
\hline 2 & 277 & 71 & 258 & 216 & 298 & 286 & 69 & 256 & 236 & 285 & 276 & 71 & 250 & 237 \\
\hline 3 & 380 & 67 & 314 & 222 & 307 & 316 & 71 & 246 & 241 & 318 & 319 & 69 & 252 & 259 \\
\hline 4 & 396 & 74 & 339 & 235 & 419 & 425 & 70 & 377 & 231 & 432 & 436 & 71 & 388 & 238 \\
\hline 5 & 376 & 76 & 299 & 218 & 362 & 381 & 70 & 318 & 226 & 372 & 395 & 71 & 327 & 229 \\
\hline 6 & 421 & 70 & 309 & 217 & 368 & 378 & 73 & 271 & 239 & 350 & 365 & 70 & 273 & 244 \\
\hline 7 & 299 & 70 & 261 & 225 & 305 & 299 & 71 & 277 & 209 & 306 & 315 & 72 & 267 & 228 \\
\hline 8 & 326 & 695 & 273 & 226 & 341 & 347 & 72 & 280 & 260 & 349 & 357 & 71 & 300 & 240 \\
\hline Mean & 345 & 71 & 288 & 223 & 338 & 344 & 71 & 289 & 234 & 342 & 348 & 71 & 294 & 239 \\
\hline
\end{tabular}




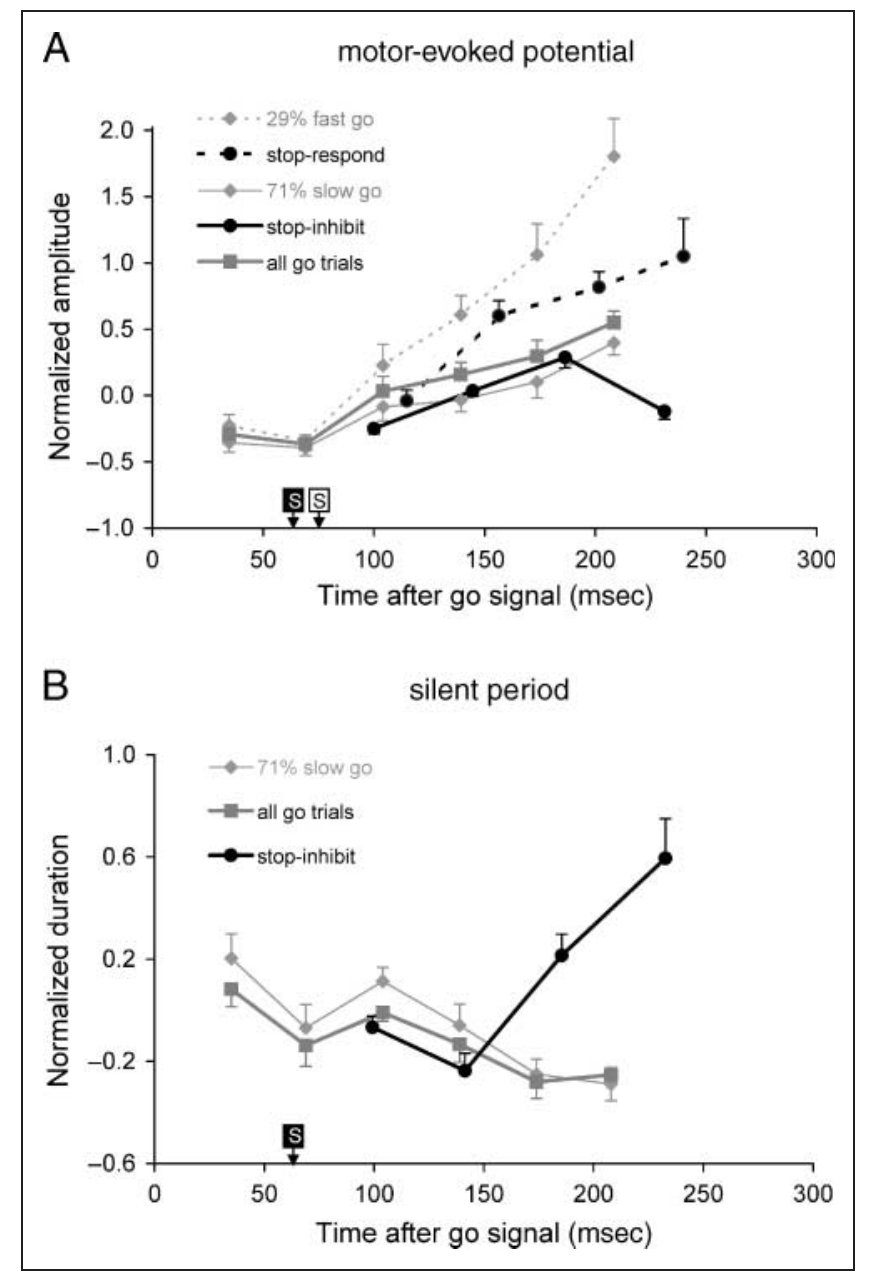

Figure 4. Normalized MEP amplitude (A) and SP duration (B) as a function of TMS interval on go trials (in gray), on stop-inhibit trials (solid black line), stop-respond (dotted black line), corresponding $29 \%$ fast go trials (dotted thin gray line), and $71 \%$ slow go trials (solid thin gray line). Zero marks go-signal onset. Average stop-signal delays for stop-inhibit and for stop-respond trials were respectively 63 and 77 msec, indicated by the white and black "S."

(sT3 vs. sT4, $p=.001$ ). Thus, although MEP amplitude, and thus, corticospinal excitability, grows progressively as the time of overt responding approaches, only at the last instance did we see a marked reduction of MEP amplitude.

Next, in an exploratory analysis of stop trial, we compared MEP amplitudes on stop-inhibit trials versus stoprespond trials, although it should be kept in mind that the amount of stop-respond trials was low (respectively $26,15,9$, and 5 trials on average for sT1 to sT4) because the tracking algorithm biased successful stopping and a considerable number of stop-respond trials was rejected because of EMG activity associated with the button-press response. This comparison indicated that MEP amplitudes on stop-respond trials were significantly larger than on stop-inhibit trials, respectively 0.61 versus $-0.01[F(1$, $7)=43.99, p<.001]$. A main effect of TMS interval $[F(3$, $21)=12.35, p<.0001]$, and a significant interaction between stop trial and TMS interval $[F(3,21)=4.09, p<$ $.05]$, was also obtained. Post hoc comparisons confirmed

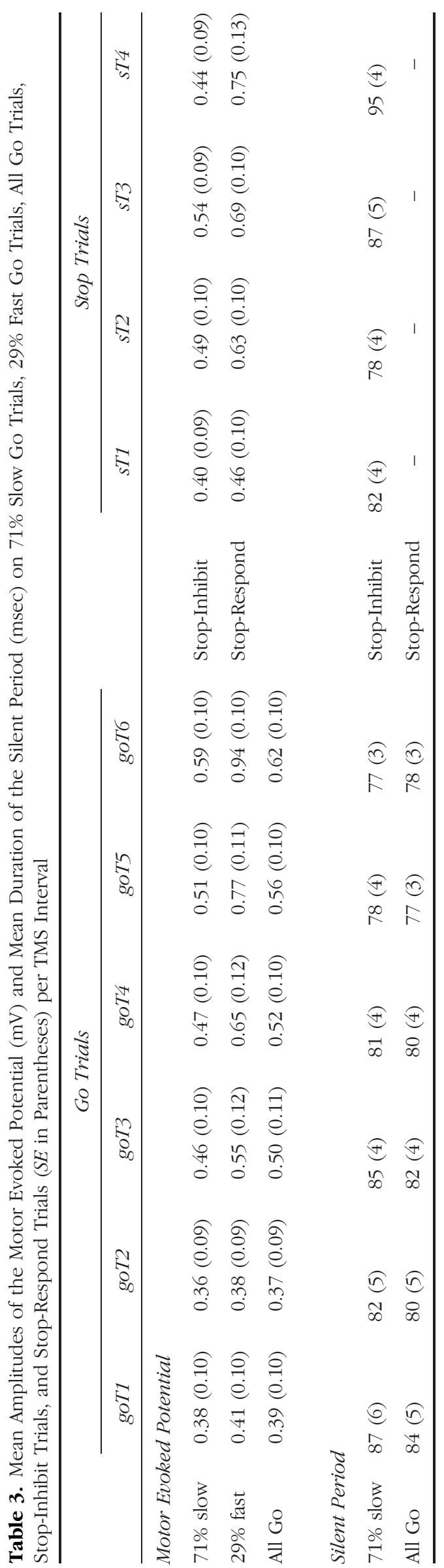


that the differences in MEP amplitude between the two stop trial types increased with TMS interval [sT2 vs. later, $F(1,7)=7.65, p<.05$, with the difference at sT4 being larger than at sT3, $F(1,7)=6.10, p<.05]$.

\section{Silent Period Duration}

\section{Go Trials}

Figure 4B (thick gray line) shows the duration of the SP expressed in $Z$-scores, aligned to the onset of the go signal. Repeated measures ANOVA yielded a significant main effect of TMS interval on the duration of the SP $[F(5,35)=$ $3.73, p=.008$ ], and confirmed a pattern of linear decrease across the six TMS intervals (subsequently, 84, 80, 82, 80, 77 , and $78 \mathrm{msec})[F(1,7)=6.07, p<.05]$

\section{Stop Trials}

Of interest are the SP data obtained from stop trials on which the response was successfully inhibited. An overall analysis yielded a significant main effect of TMS interval on the duration of the SP $[F(3,21)=15.14, p<.001]$. Figure $4 \mathrm{~B}$ (black line) shows an initial decrease in the duration of the SP between sT1 (82 msec) and sT2 (78 msec) $[F(1,7)=2.40, p<.05]$. With later stimulation on stopinhibit trials, the SP is progressively prolonged, going from $78 \mathrm{msec}$ (sT2) and $87 \mathrm{msec}$ (sT3) up to $95 \mathrm{msec}$ (sT4) $[F(2$, 14) $=14.99, p<.001]$.

Further analyses were performed to investigate the magnitude of the SP prolongation observed on stop-inhibit trials (especially at the longest TMS interval). A meaningful but conservative comparison in this respect is to directly test this value (95 $\mathrm{msec}$ ) against the SP obtained in the shortest TMS interval on go trials ( $84 \mathrm{msec}$ ) as an index of the longest SP associated with processing of the go signal. The one-tailed $t$ test yielded a significant difference between the two values $[t(7)=2.69, p=.016]$, indicating that the lengthening of the SP on successfully stopped trials extends well beyond baseline levels. This lengthening supports the notion of active inhibition upon a stop signal and argues against a sheer return to baseline. On stop-respond trials, the EMG of the voluntary response very often interrupted the SP, leading to only three trials for sT3 and only two observations for sT4, on average. Therefore, the SP on stop-respond trials was not analyzed further.

\section{Comparing Stop Trials with Corresponding Go Trials}

When comparing go- and stop-related activation, an analysis commonly reported in the stop literature compares stop trials with go trials that are derived from part of the go RT distribution (Logan \& Cowan, 1984). Following the assumptions of the race model, it can be assumed that $29 \%$ of the go trials to the left of the finish line, represent- ing fast go RTs, should correspond to stop-respond trials (see Figure 2). Accordingly, for the remaining $71 \%$ of the go trials, RT is too long so that the stop process wins the race and should thus correspond to stop-inhibit trials. Figure 4A confirms a high degree of MEP overlap between the last four TMS intervals on slow corresponding go trials (thin gray line) and on stop-inhibit trials (solid black line) $[F(1,7)=1.32, p=.29]$. The significant interaction between these two trial types $[F(3,21)=8.98, p=.01]$ results from the significantly reduced MEP on stop-inhibit trials at the last stimulation interval $[F(1,7)=12.82, p=$ .009]. A similar comparison between stop-respond trials (dotted black line) and corresponding fast go trials (dotted gray line) indicates that corticospinal excitability is reduced on stop-respond trials $[F(1,7)=6.37, p=.04]$. This pattern suggests that, on the proportion of trials on which participants were not able to stop their overt response, stop-signal processing, nevertheless, reduced corticospinal excitability. Figure $4 \mathrm{~B}$ shows that at sT3 and sT4, the SP on stop-inhibit trials was significantly longer than on corresponding slow go trials $[F(3,21)=$ $28.77, p<.01]$.

\section{Response Inhibition versus Withdrawal of Voluntary Drive}

Maintaining a precontraction level to generate background EMG requires voluntary drive to the corticospinal system. SP duration may possibly reflect the balance between intracortical inhibitory mechanisms and the facilitation of corticospinal neurons by voluntary drive (but see Inghilleri et al., 1993). Therefore, the observed SP lengthening on stop-inhibit trials could simply reflect the withdrawal of voluntary drive to the corticospinal system, rather than an active inhibitory process. We tested the hypothesis that the stop-related SP lengthening (partly) reflects a release of tonic activity as participants let go of the tonic contraction associated with the force window. Along this argument, time is crucial because the prolongation of the SP on stop-inhibit trials is time specific: SP increased from sT2 to sT3 and sT4, at respectively 90, 135, and $180 \mathrm{msec}$ after the stop signal. Voluntary drive was expressed as averaged EMG and force activity at sT1, sT2, sT3, and sT4, and was compared to mean activity recorded in seven windows, namely, during 50 msec before the go signal, 100-50 and 50 msec before the stop signal, 100-50 and $50 \mathrm{msec}$ before TMS, and 50-100 and 100-150 msec post-SP. Comparisons show that EMG levels after the end of the SP were increased at sT1 and sT2 relative to levels before stopsignal presentation $(p s<.01)$. Importantly, at sT3 and sT4, which are associated with increased SP duration, post-SP EMG levels were also increased compared to the precontraction level prior to the stop signal $(p s<$ $.05)$. The level of response force was also enhanced just after the SP, even for sT3 and s'T4 ( $p$ s < .0001). Thus, on stop-inhibit trials, EMG and force activation levels after 
the SP are higher compared to activation levels associated with background EMG, even at sT3 and sT4, which are associated with the longest SP. This increase does not fit with the hypothesis that participants were withdrawing voluntary drive and letting go of the precontraction force. Therefore, the SP prolongation on stop-inhibit trials cannot easily be explained in terms of a withdrawal of voluntary drive. The more plausible explanation is that the SP increase on stop-inhibit trials reflects the recruitment of intracortical inhibitory circuits through $\mathrm{GABA}_{\mathrm{B}}$ receptors (Terao \& Ugawa, 2002; Schnitzler \& Benecke, 1994) that inhibit activation related to the voluntary response that was triggered by the go signal.

\section{Motor Excitability Predicts Probability of Successful Stopping}

Analysis of MEP amplitude revealed that the excitability of the corticospinal tract was a priori higher for failed inhibition than for successful inhibition. We explored this relationship using Bayesian analyses by computing $p(S \mid E)$, the probability of stopping $(S)$ given the a priori knowledge of the excitability $(E)$. In the present situation, Bayes inference becomes: $p(S \mid E)=\frac{p(E \mid S) \cdot p(S)}{p(E)}$ where, $p(E \mid S)$ is the probability of a given level of excitability for successful stopping, $p(S)$ is the probability of stopping and $p(E)$ corresponds to the distribution of excitability of all stop trials (both stop-inhibit and stop-respond). We computed $p(S \mid E)$ for all individual subjects using MEP and SP Z-scores. The MEP and SP data were collapsed over TMS interval and split into categories of equal width in $Z$-score space. Categories were chosen in such a way that conditional probabilities could be computed for each subject. This condition was met for five SP categories but only for four MEP categories. Proportions were arcsine transformed $p^{\prime}=\arcsin \sqrt{(p)}$ before they were submitted to analysis of variance (Winer, 1970). Statistical analysis revealed that the probability of stopping decreased as MEP amplitude increased $[F(3,21)=9.43$, $p<.001 ;$ Figure 5A]. The linear component was also largely significant $[F(1,7)=58.78, p<.001]$. Similar results were obtained for the SP, as shown in Figure $5 \mathrm{~B}$. The probability of stopping decreased as SP shortened $[F(4,28)=19.16, p<.001]$, and this relation was also linear $[F(1,7)=59.70, p<.001]$.

\section{DISCUSSION}

In the present study, we applied single-pulse TMS of M1 at various intervals to track the time course of cortical and corticospinal excitability related to response inhibition in the stop-signal task (Logan, 1994). The timing of TMS was tailored to the individual's profile of responding to go signals and stopping to stop signals, respectively, go RT and SSRT. The behavioral index of stopping efficiency was determined at 239 msec and was characterized by low interindividual variation (i.e., $10 \mathrm{msec}$ ). SSRT was comparable over calibration and control sessions, implying that TMS did not influence stopping latency, and thus, provides a valid method to probe the state of the motor system. Many studies that recruited healthy adult samples report SSRTs in the lower $200 \mathrm{msec}$ range (see Logan, 1994 for a review). The value of 239 msec reported in the current study likely relates to the insertion of catch signals in the stimulus set (van den Wildenberg, van der Molen, \& Logan, 2002).

The goal was to measure the time course of changes in excitability of the motor system when manual responses are produced or inhibited. EMG from the involved hand muscles (flexor pollicis brevis) evoked by TMS was monitored to assess the excitability of the corticospinal tract as indexed by the amplitude of the MEP, and the recruitment of $\mathrm{GABA}_{\mathrm{B}}$ ergic inhibitory circuits at M1 as reflected by the duration of the cortical SP. MEP amplitudes related to response generation were characterized by a pattern of rapidly increasing corticospinal excitability, starting about $100 \mathrm{msec}$ after the presentation of the instruction signal to issue a button press (see also van Elswijk, Kleine, Overeem, \& Stegeman, 2007; Chen et al., 1998; Day et al., 1989). This pattern likely reflects increased firing
Figure 5. Probability of stopping decreases with increasing MEP amplitude (A) and shorter SP (B).

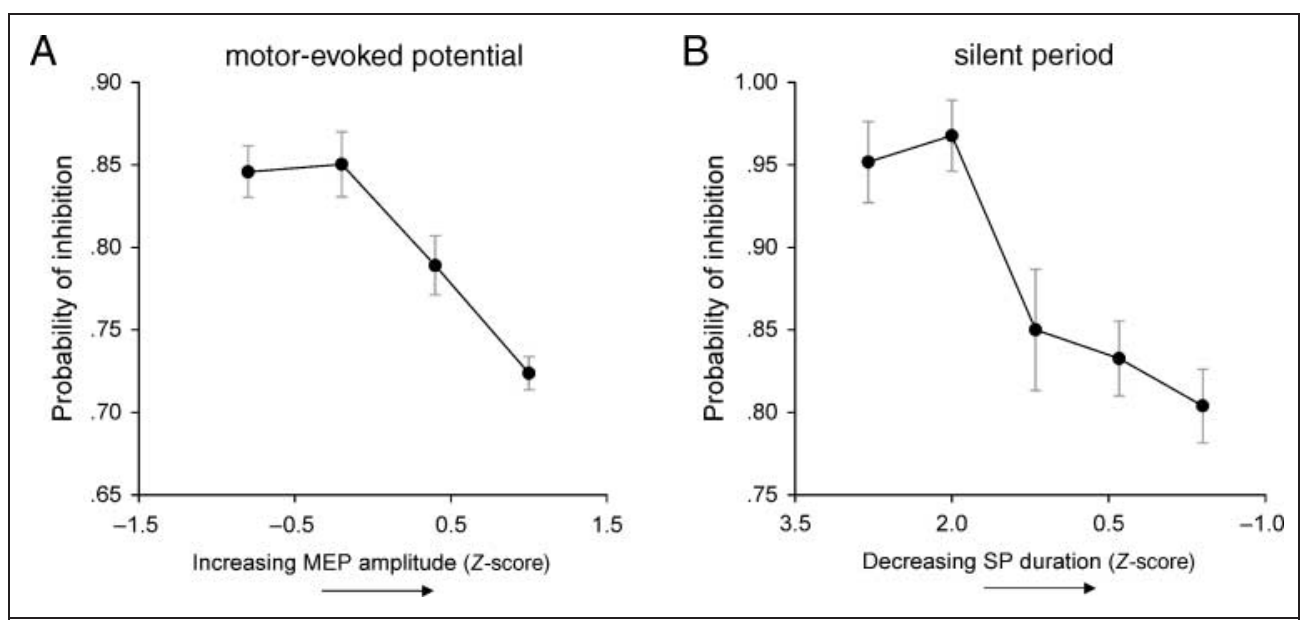


of pyramidal neurons on spinal motoneurons, thereby decreasing the excitability threshold of the pyramidal tract to facilitate the motor command (Evarts, 1966). The concurrent decrease in SP duration following go signals suggests that response generation is accompanied by a removal of intracortical inhibition, resulting in a net activation (Ni, Gunraj, \& Chen, 2007).

The timing of TMS was varied relative to the onset of the stop signal to allow tracking of the processing dynamics of the motor system in relation to stopping. A salient observation concerns the sharp decrease in MEP amplitude on successfully stopped trials, reflecting a reduction of corticospinal excitability. This observation is in line with paired-pulse MEP studies that used short intervals and that have shown that intracortical inhibitory circuits (i.e., SICI, short-interval intracortical inhibition) at M1 contribute to the suppression of activation in no-go situations (Sohn et al., 2002; Waldvogel et al., 2000) and when preventing response initiation (Coxon et al., 2006). Analyses of stopinhibit MEPs indicated an initial MEP increase related to the processing of the previously presented go signal. As depicted in Figure 4A, this transient increase of corticospinal excitability closely overlaps with motor activity derived from the $71 \%$ slow go trials that, according to the race model assumption of independence, should correspond to stop-inhibit trials (Logan \& Cowan, 1984). Comparing these two trial types, it shows that the instruction to stop responding takes about $180 \mathrm{msec}$ after stop-signal onset to counteract go-signal processing, replacing it by a pattern of decreasing corticospinal excitability.

When taken in isolation, the MEP pattern on stop-inhibit trials might be taken to suggest a mere return to baseline levels of motor activity at the longest TMS interval. However, this does not hold for the current SP findings, which point to increased excitability of $\mathrm{GABA}_{\mathrm{B}}$ ergic intracortical inhibitory neurons on stop-inhibit trials (Schnitzler \& Benecke, 1994). The SP related to stopping was characterized by a significant prolongation that was observed as early as $134 \mathrm{msec}$ after the presentation of the stop signal. This increase in SP duration at ST3 preceded the decline in MEP amplitude observed at $179 \mathrm{msec}$ (sT4), thus the increase in intracortical inhibition appears to lead the decrease in corticospinal excitability. This may suggest that the recruitment of $\mathrm{GABA}_{\mathrm{B}}$ ergic activity (the source of inhibition) is responsible for the subsequent decline in corticospinal excitability (the site of inhibition), at least in situations where the go task does not involve a choice between alternative responses. The SP prolongation as a physiological counterpart of response suppression occurred at 134 msec following a stop signal, which is about 100 msec prior to the obtained SSRT that can be viewed as the finish of the stop process.

Although the experiment was designed to obtain sufficient numbers of stop-inhibit trials, analyses of stoprespond trials provided insight into the dynamic interplay between the activation and suppression of responses. Apparently, the notion that the chances of stopping de- crease as motor excitability increases, as confirmed by Bayesian analyses, suggests a "point of no return," and that the race between go and stop processes can be rephrased in terms of the accumulation of response-related activation instead of the accumulation of time (see also Boucher, Palmeri, Logan, \& Schall, 2007).

It has been suggested that the late part of the cortical SP reflects the $\mathrm{GABA}_{\mathrm{B}}$ receptor-mediated component of the inhibitory postsynaptic potential, an intracortical inhibition mechanism similar to that of LICI when presenting paired-pulse TMS at longer intervals (Werhahn, Kunesch, Noachtar, Benecke, \& Classen, 1999; Siebner, Dressnandt, Auer, \& Conrad, 1998). However, the stop-related SP increase observed here does not seem to agree with a paired-pulse TMS study by Sohn et al. (2002), who concluded from enhanced long-interval MEPs in a no-go situation that LICI is a mechanism that is less likely to be involved in volitional inhibition of M1. Differences in experimental design might explain this discrepancy. First, although presumably mediated by a similar mechanism, LICI reported by Sohn et al. was measured by paired-pulse MEPs, whereas LICI in the present study was reflected by the duration of the SP. Second, paired-pulse TMS was applied at a fixed time following a no-go signal (i.e., around the average go RT of $290 \mathrm{msec}$ ), whereas the SP in the present study was measured at four intervals shortly after the stop signal (i.e., 45, 89, 134, and $179 \mathrm{msec}$ ), so that the dynamics of intracortical inhibition could be tracked. Third, whereas the processing of no-go signals that occur with $50 \%$ probability may not involve the LICI mechanism located at M1, the active countermanding of an ongoing response in the stop-signal paradigm might rely on stronger forms of response inhibition and does seem to recruit $\mathrm{GABA}_{\mathrm{B}}$ ergic intracortical mechanisms.

It could be argued whether a reversal of the coil constitutes a proper condition to control for peripheral effects of TMS such as subject bias and auditory clicks. Given that inhibitory interneurons have a lower threshold than excitatory interneurons (Davey et al., 1994), cortical stimulation by means of a reversed coil might preferentially recruit inhibitory cortical circuits. Although MEPs and SPs were absent in the reversed-coil condition, we cannot rule out the possibility that intracortical inhibitory interneurons were activated, which may have influenced the data collected under the control condition. This underscores the need for developing alternative control techniques to dissociate effects of brain stimulation from peripheral effects related to the clicking sound and tactile sensations that accompany TMS (see also Hoeft, Wu, Hernandez, Glover, \& Shimojo, 2008).

A limitation of the present study is that EMG recordings were restricted to the flexor pollicis brevis of the right hand. Participants responded to a go signal by pressing with their right thumb. Future stop-signal studies using TMS may extend the number of response alternatives by implementing a go task that requires a choice response with either the left or right hand. MEP and SP analyses 
then could test whether response inhibition is confined to the hand that is stopped or extends to both hands in a more general fashion. Another direction for future research is the application of paired-pulse TMS at several intervals following the stop signal. Analyses of MEP amplitudes that are triggered by paired-pulse stimulation can verify the time course of the recruitment of $\mathrm{GABA}_{\mathrm{B}}$ ergic interneurons that is indicated by the present SP findings.

In summary, we have built upon previous TMS work with go/no-go and movement-prevention paradigms, and extended that work to study the physiological mechanisms of response inhibition in the widely employed stop task. The prolongation of the SP demonstrates an active act of control, that is, an active cancellation of response processing that goes beyond a return to baseline levels of activity. By capturing the temporal dynamics of corticospinal and intracortical inhibition mechanisms, we complement structural approaches that indicated a distributed network of vital brain regions involved in inhibiting ongoing manual responses in the stop-signal paradigm (Aron et al., 2007). The results extend our knowledge on the neural basis of inhibitory control in several ways. First, a dynamic interplay between the accumulation of response activation and the attempted inhibition process was revealed at the level of M1 during stop-signal inhibition. The chances of successful response inhibition decreased progressively as a function of accumulated activation of the motor system. Second, inhibition of the motor system is expressed by an increase in excitability of $\mathrm{GABA}_{\mathrm{B}}$ ergic intracortical inhibitory circuits as early as $134 \mathrm{msec}$ after the stop signal. Second, this pattern is followed by a sharp decrease in corticospinal excitability around 180 msec following the instruction to stop an ongoing action. These dynamic patterns provide strong evidence for the accumulation of active response inhibition with an intracortical origin when stopping an ongoing response and underline the utility of the stop-signal paradigm as a tool for studying individual differences in response inhibition.

\section{Acknowledgments}

This work was supported by a grant from the Fyssen Foundation and a VENI grant from the Netherlands Organization for Scientific Research (NWO) to W. P. M. v. d. W., and a VICI grant from NWO to K. R. R. We thank Rémy Pernaud for programming the computer task and Christophe Tandonnet and Birte U. Forstmann for helpful discussions. Finally, we thank two anonymous reviewers for helpful comments on previous versions of the manuscript.

Reprint requests should be sent to Wery P. M. van den Wildenberg, Amsterdam Center for the Study of Adaptive Control in Brain and Behavior (Acacia), Department of Psychology, University of Amsterdam, Roetersstraat 15, 1018 WB, Amsterdam, The Netherlands, or via e-mail: w.p.m.vandenwildenberg@uva.nl.

\section{Note}

1. Stop-signal RT did not distinguish between the calibration session, control session, and TMS session $(p>.05)$.

\section{REFERENCES}

Aron, A. R., Behrens, T. E., Smith, S., Frank, M. J., \& Poldrack, R. A. (2007). Triangulating a cognitive control network using diffusion-weighted magnetic resonance imaging (MRI) and functional MRI. Journal of Neuroscience, 27, 3743-3752.

Aron, A. R., Fletcher, P. C., Bullmore, E. T., Sahakian, B. J., \& Robbins, T. W. (2003). Stop signal inhibition disrupted by damage to right inferior frontal gyrus in humans. Nature Neuroscience, 6, 115-116.

Aron, A. R., \& Poldrack, R. A. (2005). The cognitive neuroscience of response inhibition: Relevance for genetic research in attention-deficit/hyperactivity disorder. Biological Psychiatry, 57, 1285-1292.

Aron, A. R., \& Poldrack, R. A. (2006). Cortical and subcortical contributions to stop signal response inhibition: Role of the subthalamic nucleus. Journal of Neuroscience, 26, 2424-2433.

Aron, A. R., Robbins, T. W., \& Poldrack, R. A. (2004). Inhibition and the right inferior frontal cortex. Trends in Cognitive Sciences, 8, 170-177.

Band, G. P. H., \& van Boxtel, G. J. M. (1999). Inhibitory motor control in stop paradigms: Review and reinterpretation of neural mechanisms. Acta Psychologica, 101, 179-211.

Band, G. P. H., van der Molen, M. W., \& Logan, G. D. (2003). Horse race model simulations of the stop-signal procedure. Acta Psychologica, 112, 105-142.

Boucher, L., Palmeri, T. J., Logan, G. D., \& Schall, J. D. (2007). Inhibitory control in mind and brain: An interactive race model of countermanding saccades. Psychological Review, 114, 376-397.

Burle, B., Bonnet, M., Vidal, F., Possamaï, C.-A., \& Hasbroucq, T. (2002). A transcranial magnetic stimulation study of information processing in the motor cortex: Relationship between the silent period and reaction time. Psychophysiology, 39, 207-217.

Casey, B. J., Forman, S. D., Franzen, P., Berkowitz, A., Braver, T. S., Nystrom, L. E., et al. (2001). Sensitivity of prefrontal cortex to changes in target probability: A functional MRI study. Human Brain Mapping, 13, 26-33.

Casey, B. J., Tottenham, N., \& Fossella, J. (2002). Clinical, imaging, lesion, and genetic approaches toward a model of cognitive control. Developmental Psychobiology, 40, 237-254.

Chambers, C. D., Bellgrove, M. A., Stokes, M. G., Henderson, T. R., Garavan, H., Robertson, I. H., et al. (2006). Executive "brake failure" following deactivation of human frontal lobe. Journal of Cognitive Neuroscience, 18, 444-455.

Chen, R., Yaseen, Z., Cohen, L. G., \& Hallett, M. (1998). Time course of corticospinal excitability in reaction time and self-paced movements. Annals of Neurology, 44, 317-325.

Colzato, L. S., van den Wildenberg, W. P. M., \& Hommel, B. (2007). Impaired inhibitory control in recreational cocaine users. PLOS ONE, 2, e1143.

Coxon, J. P., Stinear, C. M., \& Byblow, W. D. (2006). Intracortical inhibition during volitional inhibition of prepared action. Journal of Neurophysiology, 95, 3371-3383.

Coxon, J. P., Stinear, C. M., \& Byblow, W. D. (2007). Selective inhibition of movement. Journal of Neurophysiology, 97, 2480-2489.

Davey, N. J., Romaiguère, P., Maskill, D. W., \& Ellaway, P. H. (1994). Suppression of voluntary motor activity revealed using transcranial magnetic stimulation of the motor cortex in man. Journal of Physiology, 477, 223-235.

Davranche, K., Tandonnet, C., Burle, B., Meynier, C., Vidal, F., \& Hasbroucq, T. (2007). The dual nature of time preparation: Neural activation and suppression revealed by transcranial 
magnetic stimulation of the motor cortex. European Journal of Neuroscience, 25, 3766-3774.

Day, B. L., Rothwell, J. C., Thompson, P. D., Noordhout, A., Nakashima, K., Shannon, K., et al. (1989). Delay in the execution of voluntary movement by electrical or magnetic brain stimulation in intact man. Brain, 112, 649-663.

Durston, S., Thomas, K. M., Worden, M. S., Yang, Y., \& Casey, B. J. (2002). The effect of preceding context of inhibition: An event-related fMRI study. Neuroimage, 16, 449-453.

Eimer, M. (1993). Effects of attention and stimulus probability on ERPs in a Go/Nogo task. Biological Psychology, 35, 123-138.

Evarts, E. V. (1966). Pyramidal tract activity associated with a conditioned hand movement in the monkey. Journal of Neurophysiology, 29, 1011-1027.

Fillmore, M. T., \& Rush, C. R. (2002). Impaired inhibitory control of behavior in chronic cocaine users. Drug and Alcobol Dependence, 6, 265-273.

Fuhr, P., Cohen, L. G., Roth, B. J., \& Hallett, M. (1991) Latency of motor evoked potentials to focal transcranial magnetic stimulation varies as a function of scalp positions stimulated. Clinical Neurophysiology, 81, 81-89.

Garavan, H., Ross, T. J., \& Stein, E. A. (1999). Right hemispheric dominance of inhibitory control: An event-related functional MRI study. Proceedings of the National Academy of Sciences, U.S.A., 96, 8301-8306.

Gauggel, S., Rieger, M., \& Feghoff, T. A. (2004). Inhibition of ongoing responses in patients with Parkinson's disease. Journal of Neurology: Neurosurgery and Psychiatry, 75, 539-544.

Hallett, M. (2007). Transcranial magnetic stimulation: A primer. Neuron, 55, 187-199.

Hoeft, F., Wu, D.-A., Hernandez, A., Glover, G. H., \& Shimojo, S. (2008). Electronically switchable sham transcranial magnetic stimulation (TMS) system. PLOS ONE, 3, e1923.

Hoshiyama, M., Kakigi, R., Koyama, S., Takeshima, Y., Watanabe, S., \& Shimojo, M. (1997). Temporal changes of pyramidal tract activities after decision of movement: A study using transcranial magnetic stimulation of the motor cortex in humans. Electroencephalograpby and Clinical Neurophysiology, 105, 255-261.

Hoshiyama, M., Koyama, S., Kitamura, Y., Watanabe, S. Shimojo, M., \& Kakigi, R. (1996). Effects of judgement process on motor evoked potentials in $\mathrm{Go} / \mathrm{No}$-go hand movement task. Neuroscience Research, 24, 427-430.

Inghilleri, M., Berardelli, A., Cruccu, G., \& Manfredi, M. (1993). Silent period evoked by transcranial stimulation of the human cortex and cervicomedullary junction. Journal of Pbysiology, 466, 521-534.

Jasper, H. H. (1958). The 10-20 electrode system of the International Federation. Clinical Neuropbysiology, 10, 371-375.

Jodo, E., \& Kayama, Y. (1992). Relation of a negative ERP component to response inhibition in a go/no-go task. Electroencephalography and Clinical Neurophysiology, 2, 477-482.

Kelly, A. M. C., Hester, R., Murphy, K., Javitt, D. C., Foxe, J. J., \& Garavan, H. (2004). Prefrontal-subcortical dissociations underlying inhibitory control revealed by event-related fMRI. European Journal of Neuroscience, 19, 3105-3112.

Kok, A. (1986). Effects of degradation of visual stimulation on components of the event-related potential (ERP) in Go/NoGo reaction tasks. Biological Psychology, 23, 21-38.

Kok, A., Ramautar, J., de Ruiter, M., \& Ridderinkhof, K. R. (2004). ERP components associated with successful and unsuccessful stopping in a stop-signal task. Psychophysiology, 41, 9-20.
Kujirai, T., Caramia, M. D., Rothwell, J. C., Day, B. L., Thompson, P. D., Ferbert, A., et al. (1993). Corticocortical inhibition in human motor cortex. Journal of Pbysiology, 471, 501-519

Leocani, L., Cohen, L. G., Wassermann, E. M., Ikoma, K., \& Hallett, M. (2000). Human corticospinal excitability evaluated with transcranial magnetic stimulation during different reaction time paradigms. Brain, 123, 1161-1173.

Levitt, H. (1971). Transformed up-down methods in psychoacoustics. Journal of the Acoustical Society of America, 49, 467-477.

Li, C.-S. R., Krystal, J. H., \& Mathalon, D. H. (2005). Fore-period effect and stop signal reaction time. Experimental Brain Research, 167, 305-309.

Logan, G. D. (1994). On the ability to inhibit thought and action: A users' guide to the stop signal paradigm. In D. Dagenbach \& T. H. Carr (Eds.), Inbibitory processes in attention, memory, and language (pp. 189-239). San Diego, CA: Academic Press.

Logan, G. D., \& Cowan, W. B. (1984). On the ability to inhibit thought and action: A theory of an act of control. Psychological Review, 91, 295-327.

Nakata, H., Inui, K., Wasaka, T., Tamura, Y., Akatsuka, K., Kida, T., et al. (2006). Higher anticipated force required a stronger inhibitory process in go/nogo tasks. Clinical Neurophysiology, 117, 1669-1676.

Nakata, H., Inui, K., Wasaka, T., Tamura, Y., Kida, T., \& Kakigi, R. (2005). Effects of ISI and stimulus probability on event-related go/nogo potentials after somatosensory stimulation. Experimental Brain Research, 62, 293-299.

Ni, Z., Gunraj, C., \& Chen, R. (2007). Short interval intracortical inhibition and facilitation during the silent period in human. Journal of Physiology, 583, 971-982.

Nickerson, R. S. (1973). Intersensory facilitation of reaction time: Energy summation of preparation enhancement? Psychological Review, 80, 489-509.

Nieuwenhuis, S., Yeung, N., van den Wildenberg, W. P. M., \& Ridderinkhof, K. R. (2003). Electrophysiological correlates of anterior cingulate function in a go/no-go task: Effects of response conflict and trial type frequency. Cognitive, Affective, and Behavioral Neuroscience, 3, 17-26.

Nigg, J. T. (2005). Neuropsychologic theory and findings in attention-deficit/hyperactivity disorder: The state of the field and salient challenges for the coming decade. Biological Psychiatry, 57, 1424-1435.

Penadés, R., Catalán, R., Rubia, K., Andrés, S., Salamero, M. \& Gastó, C. (2006). Impaired response inhibition in obsessive compulsive disorder. European Psychiatry, 22, 404-410.

Pfefferbaum, A., Ford, J. M., Weller, B. J., \& Kopell, B. S. (1985). ERPs to response production and inhibition. Electroencephalography and Clinical Neurophysiology, 60, 423-434.

Reis, J., Swayne, O. B., Vandermeeren, Y., Camus, M., Dimyan, M. A., Harris-Love, M., et al. (2008). Contribution of transcranial magnetic stimulation to the understanding of cortical mechanisms involved in motor control. Journal of Pbysiology, 586, 325-351.

Ridderinkhof, K. R., Scheres, A., Oosterlaan, J., \& Sergeant, J. A. (2005). Delta plots in the study of individual differences: New tools reveal response inhibition deficits in $\mathrm{AD} / \mathrm{HD}$ that are eliminated by methylphenidate treatment. Journal of Abnormal Psychology, 114, 197-215.

Rossini, P. M., Zarola, F., Stalberg, E., \& Caramia, M. (1988). Pre-movement facilitation of motor-evoked potentials in man during transcranial stimulation of the central motor pathways. Brain Research, 458, 20-30. 
Rubia, K., Russell, T., Overmeyer, S., Brammer, M. J., Bullmore, E. T., Sharma, T., et al. (2001). Mapping motor inhibition: Conjunctive brain activations across different versions of go/no-go and stop tasks. Neuroimage, 13, 250-361.

Sanger, T. D., Garg, R. R., \& Chen, R. (2001). Interactions between two different inhibitory systems in the human cortex. Journal of Physiology, 530, 307-317.

Schachar, R., \& Logan, G. D. (1990). Impulsivity and inhibitory control in normal development and childhood psychopathology. Developmental Psychology, 26, 710-720.

Schnitzler, A., \& Benecke, R. (1994). The silent period after transcranial magnetic stimulation is of exclusive cortical origin: Evidence from isolated cortical ischemic lesions in man. Neuroscience Letters, 180, 41-45.

Siebner, H. R., Dressnandt, J., Auer, C., \& Conrad, B. (1998). Continuous intrathecal baclofen infusion induced a marked increase of the transcranially evoked silent period in a patient with generalized dystonia. Muscle and Nerve, 21, 1209-1212.

Simmonds, D. J., Pekar, J. J., \& Mostofsky, S. H. (2008). Meta-analysis of Go/No-go tasks demonstrating that fMRI activation associated with response inhibition is task-dependent. Neuropsychologia, 46, 224-232.

Slater-Hammel, A. T. (1960). Reliability, accuracy, and refractoriness of a transit reaction. Research Quarterly, 31, 217-228.

Sohn, Y. H., Wiltz, K., \& Hallett, M. (2002). Effect of volitional inhibition on cortical inhibitory mechanisms. Journal of Neurophysiology, 88, 333-338.

Stinear, C. M., Coxon, J. P., \& Byblow, W. D. (2009). Primary motor cortex and movement prevention: Where stop meets go. Neuroscience \& Biobehavioral Reviews, 33, 662-673.

Terao, Y., \& Ugawa, Y. (2002). Basic mechanisms of TMS Journal of Clinical Neurophysiology, 19, 322-343.

van den Wildenberg, W. P. M., van Boxtel, G. J. M., van der Molen, M. W., Bosch, D. A., Speelman, J. D., \& Brunia, C. H. M. (2006). Stimulation of the subthalamic region facilitates the selection and inhibition of motor responses in Parkinson's disease. Journal of Cognitive Neuroscience, 18, 626-636.

van den Wildenberg, W. P. M., van der Molen, M. W., \& Logan, G. D. (2002). Reduced response readiness delays stop-signal inhibition. Acta Psychologica, 111, 115-169.

van der Molen, M. W., \& Keuss, P. G. J. (1979). The relationship between reaction time and auditory intensity in discrete auditory tasks. Quarterly Journal of Experimental Psychology, 31, 95-102.

van Elswijk, G., Kleine, B. U., Overeem, S., \& Stegeman, D. F. (2007). Expectancy induces dynamic modulation of coticospinal excitability. Journal of Cognitive Neuroscience, 19, 121-131.

Verbruggen, F., \& Logan, G. D. (2008). Response inhibition in the stop-signal paradigm. Trends in Cognitive Sciences, 12, 418-424.

Waldvogel, D., van Gelderen, P., Muellbacher, W., Ziemann, U., Immisch, I., \& Hallett, M. (2000). The relative metabolic demand of inhibition and excitation. Nature, 406, 995-998.

Walsh, V., \& Cowey, A. (2000). Transcranial magnetic stimulation and cognitive neuroscience. Nature Reviews Neuroscience, 1, 73-79.

Werhahn, K. J., Kunesch, E., Noachtar, S., Benecke, R., \& Classen, J. (1999). Differential effects on motorcortical inhibition induced by blockade of GABA uptake in humans. Journal of Physiology, 517, 591-597.

Williams, B. R., Ponesse, J. S., Schachar, R. J., Logan, G. D., \& Tannock, R. (1999). Development of inhibitory control across the life span. Developmental Psychology, 35, 205-213.

Winer, B. J. (1970). Statistical principles in experimental design. New York: McGraw-Hill.

Wylie, S. A., van den Wildenberg, W. P. M., Ridderinkhof, K. R., Bashore, T. R., Powell, V. D., Manning, C. A., et al. (2009). The effect of Parkinson's disease on interference control during action selection. Neuropsychologia, 47, 145-157.

Ziemann, U., Lonnecker, S., Steinhoff, B. J., \& Paulus, W. (1996). The effect of lorazepam on the motor cortical excitability in man. Experimental Brain Research, 109, 127-135. 
Copyright of Journal of Cognitive Neuroscience is the property of MIT Press and its content may not be copied or emailed to multiple sites or posted to a listserv without the copyright holder's express written permission. However, users may print, download, or email articles for individual use. 